Original Article

\title{
The Utility of a 3D Endoscope and Robot-Assisted System for MIDCAB
}

Yuki Endo, MD, Yoshitsugu Nakamura, MD, PhD, Miho Kuroda, MD, Yujiro Ito, MD, and Takaki Hori, MD, $\mathrm{PhD}$

\begin{abstract}
Background: Minimally invasive direct coronary artery bypass (MIDCAB) has been revived with new techniques and hybrid procedures for MIDCAB and percutaneous coronary intervention (PCI). We reviewed the midterm results of MIDCAB with a three-dimensional (3D) endoscope in our institution.

Methods: Of the 359 patients who underwent off-pump coronary artery bypass grafting (CABG) from December 2013 to March 2017, 54 had MIDCAB with the left internal thoracic artery (LITA) to left anterior descending (LAD) artery through a small left thoracotomy with a 3D endoscope. The same intercostal space was used for the main surgical incision and the insertion site of the 3D endoscope. In all, 22 patients had hybrid coronary revascularization (HCR), combined PCI and MIDCAB.

Results: There was no operative death. One patient had cerebral infarction without disability. No cases showed significant increases in CKMB. In all, 34 patients commenced ambulation on postoperative day 1 . The postoperative hospital stay was $9.1 \pm 5.0$ days. In total, 37 patients had coronary computed tomography (CT), and their patency of LITA was $100 \%$. In HCR, there was no mortality and major adverse cardiovascular event (MACE). Target lesion revascularization among 12 months was $1.6 \%$.

Conclusion: The midterm results of MIDCAB with 3D endoscope-assisted LITA harvesting were satisfactory. MIDCAB, including $\mathrm{HCR}$, is a good alternative for selected highrisk patients.
\end{abstract}

Keywords: minimally invasive direct coronary artery bypass, endoscope, robot-assisted system, hybrid coronary revascularization

\section{Introduction}

Improved percutaneous coronary intervention (PCI) results are a driving force behind minimally invasive

Department of Cardiovascular Surgery, Chiba-Nishi General Hospital, Matsudo, Chiba, Japan

Received: October 24, 2018; Accepted: January 12, 2019 Corresponding author: Yoshitsugu Nakamura, MD, PhD, Department of Cardiovascular Surgery, Chiba-Nishi General Hospital,107-1 Kanegasaku, Matsudo, Chiba, 270-2251, Japan Email: ystgnkmr@gmail.com

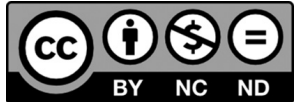

This work is licensed under a Creative Commons Attribution-NonCommercialNoDerivatives International License.

(C)2019 The Editorial Committee of Annals of Thoracic and Cardiovascular Surgery cardiac surgery (MICS) or hybrid revascularization. In addition, improving the resolution of coronary computed tomography (CT) provides information on buried coronary, chronic total occlusion (CTO), and laterality, among other parameters. However, traditional methods have the risk of damaging the rib and nerve, have a large surgical site, and have low visibility. As a solution, we used a threedimensional (3D) endoscope and instruments with MICS to harvest the left internal thoracic artery (LITA). Additionally, we used only one intercostal space, which enabled pain control and avoided damage to the rib and nerve.

\section{Pre-operation}

With axial and 3D construction of the coronary CT, the position and running of the LITA and left anterior 


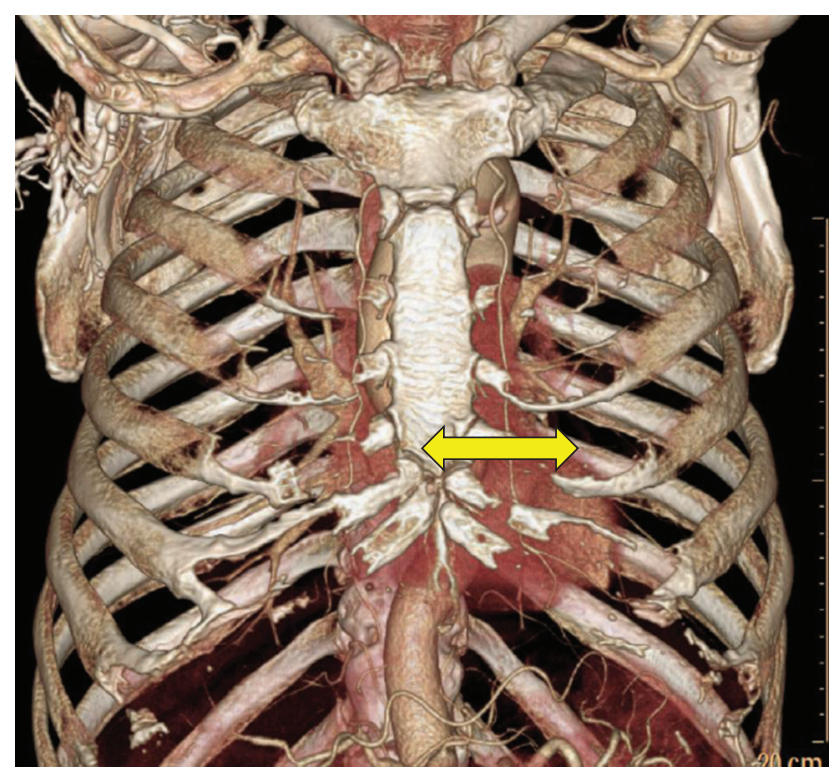

Fig. 1 Coronary CT shows how long it is away from patient's breastbone. CT: computed tomography

descending (LAD) artery were established. We measured the LAD anastomosis position from the sternum and determined the incision position (Fig. 1). At the time of surgery, after the introduction of general anesthesia, intubation with a double lumen tube was performed, and intraoperative oxygenation was performed with single lung ventilation. The towel was placed under the left shoulder blade and rotated 30 degrees to the right of the bed. The left hand was placed on the operating table and marked on the intercostal space (4th or 5th intercostal), which was decided by preoperative CT.

\section{Operation technique}

We wore 3D glasses on top of magnifying glasses during surgery. All minimally invasive direct coronary artery bypass (MIDCAB) patients underwent limited left anterolateral thoracotomy through the fourth or fifth intercostal space, and the intercostal space of the main surgical site and endoscope's insertion site were the same. A 3D endoscope and extended electrocautery device were used for the LITA harvest. When using a 3D endoscope, we used 14 Fr trockers routinely (Fig. 2a). After the LITA harvest, we confirmed that there was no bleeding from the internal thoracic artery (ITA) bed, and heparinization was performed after confirming that there was no problem with hemostasis. After pericardiotomy, we were careful not to confuse diagonal branches, and the LAD was fixed with Octopus NUVO (Medtronic, Minneapolis, MN, USA). LITA to LAD anastomosis was performed using 8-0 prolene sutures on the beating heart with Octopus NUVO,
Table 1 The patient's characteristics data for MIDCAB

\begin{tabular}{ll} 
Age & $69.8 \pm 11.7$ \\
Gender (male) & 45 \\
Hypertension & 33 \\
Hyperlipidaemia & 27 \\
Diabetes melitus & Oral:14 insulin: 4 \\
Emergency & 1 \\
Chronic kidney disease & 8 (HD: 2$)$ \\
EF & $62.4 \pm 8.6$ \\
EF<40\% & 1 \\
Euro scoreII & $3.53 \pm 1.2$ \\
\hline
\end{tabular}

EF: ejection fraction; MIDCAB: minimally invasive direct coronary artery bypass

which was inserted in the same hole with a 3D endoscope (Fig. 2b). After anastomosis, the 19Fr drain was inserted from the same insertion site with Octopus NUVO. Finally, two surgical sites, namely, the main surgical site and the drain hole, were used, and only one intercostal space was used. As a result, only one intercostal space could be used.

\section{Subjects and Methods}

Table 1 provides the patient background data. Of the 359 patients who underwent off-pump coronary artery bypass grafting (CABG) from December 2013 to August 2017, 54 (male 45, 83.3\%) had MIDCAB with LITA to LAD through a small left thoracotomy. There were 53 cases after excluding one case of midline sternotomy incision conversion. For the LITA harvest, we used 3D endoscopy for 43 cases $(81.1 \%)$ and the da Vinci Surgical System for 10 cases $(18.9 \%)$. The fourth intercostal approach was used for 46 cases $(86.8 \%)$, and the fifth intercostal approach was used for six cases $(13.2 \%)$. The average age of the patients was $69.8 \pm 11.7$ years, and an emergency operation was performed in 1 case. The ejection fraction (EF) was $62.4 \pm 8.6 \%$, one case had a low core function $(\mathrm{EF}<40 \%)$, and the Euro score II was $3.53 \pm$ 1.2 points. In terms of coronary pathology, there were 30 patients (56.6\%) with LAD single vessel disease (SVD). The coronary pathology was as follows: CTO, 13 pts; left main lesion, 8 pts; in-stent restenosis, 8 pts; and metal allergy, 1 pt. There were nine patients (16.9\%) with double vessel disease (DVD), and there were 14 (26.4\%) patients with triple vessel disease (TVD) (Fig. 3). The SYNTAX score of SVD was $18.7 \pm 6.7$ points, and the DVD and TVD scores were $35.0 \pm 11.0$ points. Eight patients had chronic kidney disease (CKD), two patients had CKD on hemodialysis, five patients had malignant tumors (lung cancer, colon cancer, and esophageal cancer), 


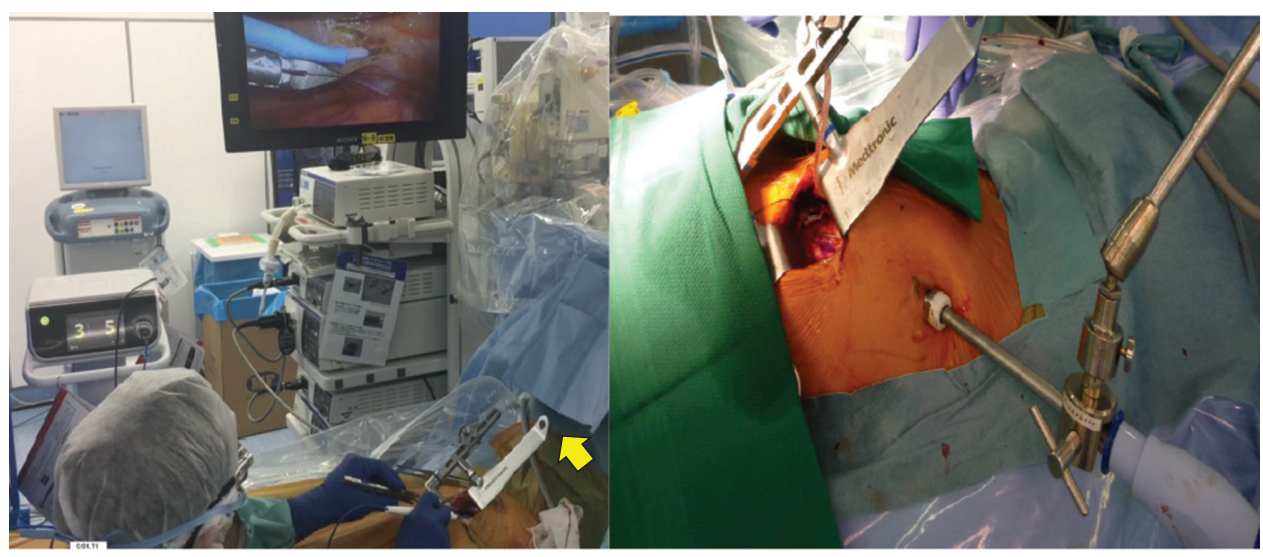

Fig. 2 (a) The surgeon harvests LITA while seeing 3D endoscope. Yellow arrow shows that the surgeon does not need to lift Thoratrack. (b) At the time of anastomosis, Octopus NUVO is inserted from the place where the endoscope was inserted. LITA: left internal horacic artery

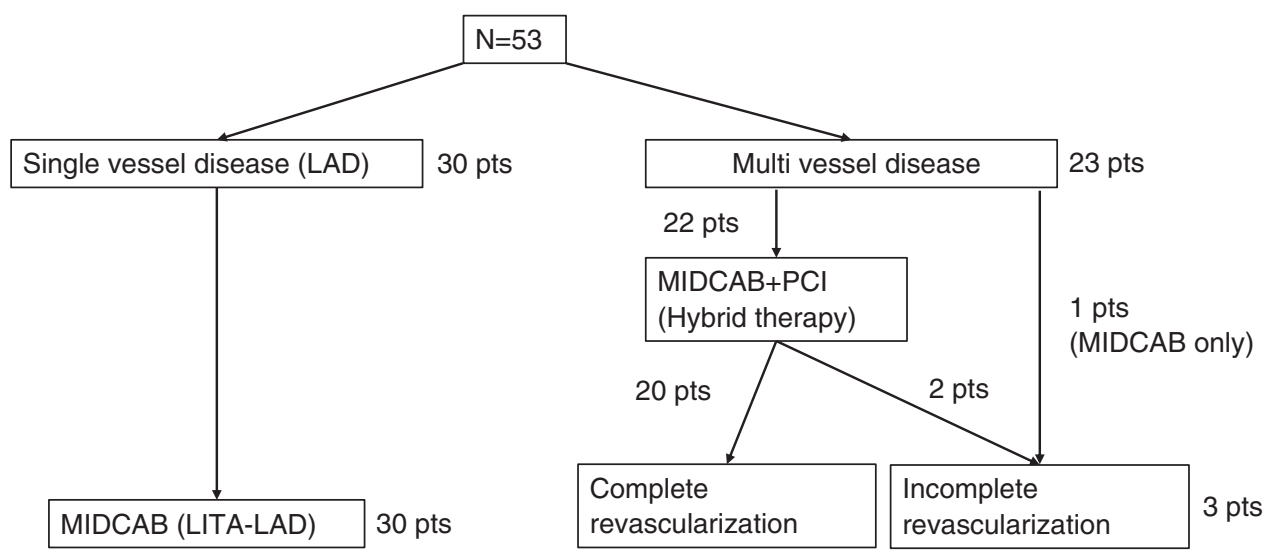

Fig. 3 The patients $(\mathrm{N}=53)$ were sorted as follows. LITA: left internal horacic artery; MIDCAB: minimally invasive direct coronary artery bypass; LAD: left anterior descending; PCI: percutaneous coronary intervention

three patients had abdominal aorta aneurysms, and three patients had chronic obstructive pulmonary disease (COPD). Among the 23 cases of multibranch lesions (DVD and TVD), one patient was enforced only by MIDCAB due to patient circumstances, and the procedure was terminated without complete blood circulation reconstruction. In all, 22 patients underwent hybrid coronary revascularization (HCR), which is a treatment of coronary revascularization by PCI and MIDCAB. Table 2 shows the patient characteristics for HCR. MIDCAB and PCI preceded 11 cases, respectively. The average patient age was $74.5 \pm 10.9$ years, and 20 patients $(90.9 \%)$ were men. There were two emergency cases (9.1\%), the EF was $63.4 \pm 8.2 \%$, and one case had low cardiac function $(4.5 \%)$. The reasons for choosing HCR were as follows: for nine cases, LAD was inappropriate
Table 2 The patient's characteristics data for HCR

\begin{tabular}{ll}
\hline Age & $74.5 \pm 10.9$ \\
Gender (male) & 20 \\
Emergency & 2 \\
Chronic kidney disease & 4 \\
EF & $63.4 \pm 8.2$ \\
EF $<40 \%$ & 1 \\
SYNTAX score & $35.0 \pm 11.0$ \\
\hline
\end{tabular}

EF: ejection fraction; HCR: hybrid coronary revascularization

for PCI; six cases had CABG implementation difficulty (due to a malignant tumor in three cases, a high midline incision risk in two cases, and a porcelain aorta in one case), and seven cases chose the procedure. After PCI, DAPT was started. Prior PCI cases were stopped before MIDCAB, and heparinization was performed. APTT was controlled for 50-70 s. 


\section{Results}

There were no deaths. No major adverse cardiovascular events (MACEs) occurred. One patient had cerebral infarction without disability, two patients had respiratory failure, and two patients had superficial wound infections. The operation time was $147 \pm 19$ minutes, and the LITA harvesting time was $46 \pm$ minutes. There was no graft injury in any patient. Five patients were extubated in the operating room, and the others were extubated in the ICU $8.0 \pm 7.4$ hours postoperation. CKMB did not increase significantly. In all, 34 patients $(83 \%)$ commenced ambulation on postoperative day 1 . The duration of the postoperative hospital stay was $9.1 \pm 5.0$ days. In total, 48 patients (91\%) had coronary CT, and their patency of LITA was $100 \%$. The average follow-up period was $12 \pm 9$ months, and one patient died due to senile decay. There were no adverse events with the $3 \mathrm{D}$ endoscope and no injuries to the LITA. In addition, there were no cases in which ITA could be observed with the scope.

The HCR results showed that 20 of the 22 patients (90.9\%) underwent complete revascularization. We performed PCI at a total of 41 locations and used drug-eluting stents (DES) for all surgeries. PCI was performed in all nine locations in the LMT (left main trunk), 15 of 17 locations in the LCX (left circumflex artery), and 17 of 18 locations in the RCA (right coronary artery). There were no deaths or cases of MACE. One patient had in-stent restenosis, and target lesion revascularization (TLR), including LAD revascularization, at 12 months was $1.6 \%$.

\section{Discussion}

MIDCAB by left small thoracotomy was first performed in 1967. This surgical method is not widely used not only due to difficulties in deployment and anastomosis but also due to aspects regarding medical equipment and technology. However, due to the development of coronary CT, grasping properties such as calcification of the LAD and the running and anastomotic position and deciding the thoracotomy position accurately became possible. At the same time, evaluating whether the LITA was running and graftable was possible, and MIDCAB became a more established surgical method.

Compared to PCI, MIDCAB is superior to LAD revascularization over a long period of time due to its good graft patency with regard to LAD single-branch lesions, and MIDCAB can prevent MACE, including restenosis in the stent. ${ }^{1,2)}$ Therefore, MIDCAB is considered to be superior to PCI, especially for patients with diffuse LAD stenosis. There are also reports ${ }^{3)}$ showing the usefulness of HCR for multibranch lesions, and for cardiovascular surgery in which minimally invasive surgery is prevalent, MIDCAB is a useful surgical method.

There are three methods for collecting the LITA in MIDCAB: (1) the lifting type (direct vision); (2) the endoscope (two-dimensional (2D) or 3D); and (3) the robotassisted surgery system. The lifting type is a conventional method, namely, a method for lifting the main wound with a retractor on the left side of the thorax and harvesting the LITA under direct vision. With this method, developing the field of view, especially on the proximal and distal part of the LITA, is difficult when harvesting the LITA, which leads to insufficient harvesting of the LITA, causing branches of the LITA to leave at the proximal part, which may result in the so-called steal syndrome. ${ }^{4)}$ Moreover, due to the poor visual field of the proximal side, excessive tension is applied to the wound by forcible lifting, which leads to postoperative pain and delayed wound healing. On the other hand, with the method using a 3D endoscope or robot-assisted surgery system, although the number of wounds itself increases, these methods enable an easier harvesting of the LITA due to a better visual field and prevent the application of excessive tension. In addition, postoperative pain can be reduced.5) Particularly, in extremely obese patients, detaching the LITA is difficult because of the large among of adipose tissue and difficulties in lifting the rib cage. However, by using an endoscope or a robot to assist in surgery, these problems are resolved to some extent. The major difference between endoscopy and surgical assistance with robots is economical. There are reports $\left.{ }^{6}\right)$ that cardiac surgery with a robotassisted surgery system requires $\$ 1$ million to $\$ 2.5$ million (US \$) per patient, and there are restrictions in terms of the facilities that can use this approach. In the case of endoscopy, either a 2D or 3D endoscope is used; however, the 3D endoscope can obtain a stereoscopic image with higher resolution than the $2 \mathrm{D}$ endoscope and grafting can be performed in a shorter time with the $3 \mathrm{D}$ endoscope. ${ }^{7)}$ Therefore, we used a 3D endoscope at our facility.

In our institution, developing the visual of the LITA proximal side and branch processing by using the $3 \mathrm{D}$ endoscope as a reason for early recovery after surgery is easy because there are few postoperative complications, and the shortening of the associated operation time has been cited. Simultaneously, this approach leads to graft patency and a decrease in complications, including MACE. In addition, we reduced the number 
of wounds and reduced postoperative wound pain by using Octopus NUVO from the insertion site of the trocar and the drain penetration part after the anastomosis, with the main wound and the trocar insertion part at the same intercostal space. This approach also helped lead to early hospital discharge. Regarding HCR, although the age of the HCR group was slightly higher than that of the MIDCAB alone group, MACE was not observed, TLR was low, and the middle term results were good.

\section{Conclusions}

With the development of coronary CT, MIDCAB has become a more established surgical method. With LITA harvesting using a 3D endoscope, developing a good visual field of the LITA proximal and side branch treatment was not difficult. As a result, a shortened surgical operation time and graft patency rate and early recovery after surgery, including a reduced ICU stay and postoperative hospitalization time, were possible. Postoperative wound pain system reduction can also help. MIDCAB using a $3 \mathrm{D}$ endoscope is useful.

\section{Disclosure Statement}

The authors have no conflicts of interest.

\section{References}

1) Aziz O, Rao C, Panesar SS, et al. Meta-analysis of minimally invasive internal thoracic artery bypass versus percutaneous revascularisation for isolated lesions of the left anterior descending artery. BMJ 2007; 334: 617.

2) Drenth DJ, Veeger NJ, Winter JB, et al. A prospective randomized trial comparing stenting with offpump coronary surgery for high-grade stenosis in the proximal left anterior descending coronary artery: three-year follow-up. J Am Coll Cardiol 2002; 40: 1955-60.

3) Halkos ME, Vassiliades TA, Myung RJ, et al. Sternotomy versus nonsternotomy LIMA-LAD grafting for single-vessel disease. Ann Thorac Surg 2012; 94: 1469-77.

4) Hrapkowicz T, Bisleri G. Endoscopic harvesting of the left internal mammary artery. Ann Cardiothorac Surg 2013; 2: 565-9.

5) Bucerius J, Metz S, Walther T, et al. Endoscopic internal thoracic artery dissection leads to significant reduction of pain after minimally invasive direct coronary artery bypass graft surgery. Ann Thorac Surg 2002; 73: 1180-4.

6) Yanagawa F, Perez M, Bell T, et al. Critical outcomes in nonrobotic vs robotic-assisted cardiac surgery. JAMA Surg 2015; 150: 771-7.

7) Tomita S, Iino K, Higashidani K, et al. Totally endoscopic off-pump coronary artery bypass surgery. J Jpn Coll Angiol 2007; 47: 65-70. 